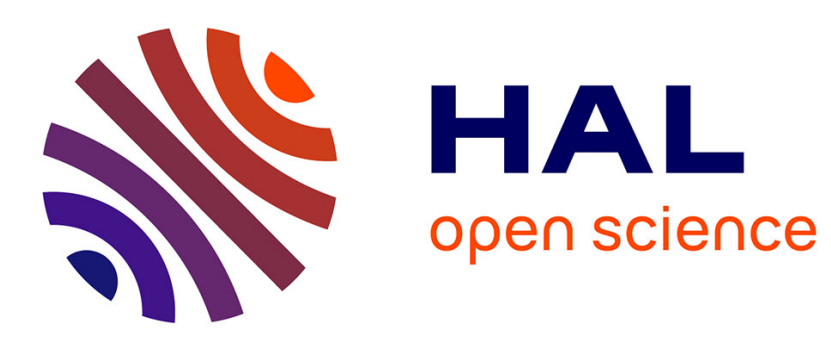

\title{
Periodic solutions of Evolution Variational Inequalities: a method of guiding functions
}

\author{
Samir Adly, Daniel Goeleven, Michel Théra
}

\section{To cite this version:}

Samir Adly, Daniel Goeleven, Michel Théra. Periodic solutions of Evolution Variational Inequalities: a method of guiding functions. Chinese Annals of Mathematics - Series B, 2009, 30 (3), pp.261-272. hal-01900177

\section{HAL Id: hal-01900177 \\ https://hal-unilim.archives-ouvertes.fr/hal-01900177}

Submitted on 21 Oct 2018

HAL is a multi-disciplinary open access archive for the deposit and dissemination of scientific research documents, whether they are published or not. The documents may come from teaching and research institutions in France or abroad, or from public or private research centers.
L'archive ouverte pluridisciplinaire HAL, est destinée au dépôt et à la diffusion de documents scientifiques de niveau recherche, publiés ou non, émanant des établissements d'enseignement et de recherche français ou étrangers, des laboratoires publics ou privés. 


\title{
Periodic Solutions of Evolution Variational Inequalities — a Method of Guiding Functions ${ }^{* * *}$
}

\author{
Samir ADLY* Daniel GOELEVEN** Michel THÉRA*
}

\begin{abstract}
This paper focuses on a part of the presentation given by the third author at the Shanghai Forum on Industrial and Applied Mathematics (Shanghai 2006). It is related to the existence of a periodic solution of evolution variational inequalities. The approach is based on the method of guiding functions.
\end{abstract}

Keywords Variational inequality, Differential inclusion, Topological degree, Guiding function, Periodic solution

2000 MR Subject Classification 49J40, 49J20, 35K85

\section{Introduction}

Variational inequalities provide a broad unifying setting for the study of optimization and equilibrium problems which have their origin in various areas such as economics and engineering. The theory of variational inequalities started around 40 years ago in two different areas: partial differential systems with G. Stampacchia and his collaborators and mathematical programming with R. Cottle.

Infinite-dimensional variational inequalities were developed for studying free boundary problems defined by non-linear partial differential equations arising mostly in unilateral mechanics. They have numerous applications as it is well-known. Let us mention for instance the books by Baiocchi and Capello [2] and Kinderleherer and Stampacchia [12] for the abstract theory and by Glowinski, Lions and Trémolière [9] for the numerical analysis. The reader is also referred to the recent book by Giannessi [8] and the references therein. The well-known generalization by Stampacchia of the Lax-Milgram lemma for coercive bilinear forms to convex sets, published in Comptes Rendus de l'Académie des Sciences in 1964 (see [20]), is the starting point of the theory of infinite-variational inequalities. This famous result was extended some years later by Lions and Stampacchia [15] to not necessarily coercive bilinear forms and has an important application in the theory of elliptic and parabolic operators and in problems with unilateral

\footnotetext{
*Département de Mathématiques et Informatique, Laboratoire XLIM (Unité Mixte 6172 du CNRS), Université de Limoges, 123 Avenue A. Thomas, 87060 Limoges Cedex, France.

E-mail: samir.adly@unilim.fr michel.thera@unilim.fr

** Laboratoire d' Informatique et de Mathématiques, Université de La Réunion, 97400 Saint-Denis, France. E-mail: daniel.goeleven@univ-reunion.fr

*** Project supported by the Australian Research Council (No. DP077014) and the STIC-AMSUD Program "Optimisation Énergétique".
} 
constraints (Signorini's problem for example). We would like to mention the abstract regularity theorem for variational inequalities associated to nonlinear monotone operators obtained by Brezis and Stampacchia [3] which applies to the case of a convex set defined via an obstacle from above and an obstacle from below.

The theory of finite-dimensional variational inequalities is related to finite-dimensional optimization and in particular to nonlinear programming. It was developed independently by the mathematical programming community first by Cottle [6] and later by several authors. The reader could find a nice survey on finite-dimensional variational inequalities and the references therein in the book by Facchinei and Pang [7]. We would like to mention particularly the work by Robinson [18] in connection with generalised equations, who developed an original framework to obtain qualitative and numerical results for variational inequalities in analogy with classical Newton-type methods.

This note focuses on a specific part of a general talk given at the Shanghai Forum on Applied and Industrial Mathematics in May 2006 and is related to a previous work of the same authors (see [1]). It concerns the existence of a $T$-periodic solution $u \in C^{0}\left([0, T] ; \mathbb{R}^{n}\right)$ of the evolution variational inequality:

$$
\frac{\mathrm{d} u}{\mathrm{~d} t}(t)+F(u(t))-f(t) \in-\partial \varphi(u(t)), \quad \text { a.e. } t \in[0, T] .
$$

In this problem the solution $u$ satifies

$$
\begin{aligned}
& \frac{\mathrm{d} u}{\mathrm{~d} t} \in L^{\infty}\left(0, T ; \mathbb{R}^{n}\right), \\
& u \text { is right-differentiable on }[0, T), \\
& u(0)=u(T) .
\end{aligned}
$$

We suppose that $F: \mathbb{R}^{n} \rightarrow \mathbb{R}^{n}$ is a continuous map, $\varphi: \mathbb{R}^{n} \rightarrow \mathbb{R}$ is a convex function, $f \in C^{0}\left(\left[0,+\infty\left[; \mathbb{R}^{n}\right)\right.\right.$ is such that $\frac{\mathrm{d} f}{\mathrm{~d} t} \in L_{\mathrm{loc}}^{1}\left(0,+\infty ; \mathbb{R}^{n}\right)$ and $T>0$ is a prescribed period and as it will be recalled later, $\partial \varphi$ is the convex subdifferential of $\varphi$.

The paper is organized as follows. In Section 2, we recall some materials. A particular attention is given to the Brouwer topological degree, since it will play a central role in the proof of the results. In Section 3, we recall the existence and uniqueness result by Schowalter [21], and we see how the problem of the existence of a periodic solution to the evolution problem (1.1) is equivalent to the existence of a fixed point of the Poincaré operator associated to the problem under consideration. Finally, Section 5 is devoted to the main theorem (Theorem 4.1) which proves the existence of a periodic solution, using the method of guiding functions.

\section{Brouwer Topological Degree and the Resolvent Operator $J_{\lambda}^{\varphi}$}

It is well-known that the degree theory is a powerful tool for the study of the existence of a solution to a nonlinear equation $f(x)=0$, where $f$ is a continuous function defined on the closure $\bar{\Omega}$ of a bounded subset $\Omega$ of $\mathbb{R}^{n}$, with values in $\mathbb{R}^{n}$ and such that 0 is outside the image of the boundary $\partial \Omega$ of $\Omega$. 
In the sequel, the scalar product on $\mathbb{R}^{n}$ is denoted as usual by $\langle\cdot, \cdot\rangle$ and $\|\cdot\|$ is the associated norm. For $r>0$, we note $\mathbb{B}_{r}:=\left\{x \in \mathbb{R}^{n}:\|x\|<r\right\}, \overline{\mathbb{B}}_{r}=\left\{x \in \mathbb{R}^{n}:\|x\| \leq r\right\}$ and $\partial \mathbb{B}_{r}:=\left\{x \in \mathbb{R}^{n}:\|x\|=r\right\}=\overline{\mathbb{B}}_{r} \backslash \mathbb{B}_{r}$.

If $f: \overline{\mathbb{B}}_{r} \rightarrow \mathbb{R}^{n}$ is continuous and $0 \notin f\left(\partial \mathbb{B}_{r}\right)$, then the Brouwer topological degree of $f$ with respect to $\mathbb{B}_{r}$ and 0 is well-defined (see, e.g., $\left.[16,17]\right)$ and is denoted by $\operatorname{deg}\left(f, \mathbb{B}_{r}, 0\right)$.

Let us now recall some properties of the topological degree that we will use later.

Proposition 2.1 (1) If $0 \notin f\left(\partial \mathbb{B}_{r}\right)$ and $\operatorname{deg}\left(f, \mathbb{B}_{r}, 0\right) \neq 0$, then there exists $x \in \mathbb{B}_{r}$ such that $f(x)=0$.

(2) Let $\varphi:[0,1] \times \overline{\mathbb{B}}_{r} \rightarrow \mathbb{R}^{n},(\lambda, x) \rightarrow \varphi(\lambda, x)$, be continuous such that, for each $\lambda \in[0,1]$, one has $0 \notin \varphi\left(\lambda, \partial \mathbb{B}_{r}\right)$. Then the map $\lambda \rightarrow \operatorname{deg}\left(\varphi(\lambda, \cdot), \mathbb{B}_{r}, 0\right)$ is constant on $[0,1]$.

(3) Let us denote by $\operatorname{id}_{\mathbb{R}^{n}}$ the identity mapping on $\mathbb{R}^{n}$. We have

$$
\operatorname{deg}\left(\operatorname{id}_{\mathbb{R}^{n}}, \mathbb{B}_{r}, 0\right)=1
$$

(4) If $0 \notin f\left(\partial \mathbb{B}_{r}\right)$ and $\alpha>0$, then

$$
\operatorname{deg}\left(\alpha f, \mathbb{B}_{r}, 0\right)=\operatorname{deg}\left(f, \mathbb{B}_{r}, 0\right)
$$

and

$$
\operatorname{deg}\left(-\alpha f, \mathbb{B}_{r}, 0\right)=(-1)^{n} \operatorname{deg}\left(f, \mathbb{B}_{r}, 0\right) .
$$

(5) If $0 \notin f\left(\partial \mathbb{B}_{r}\right)$ and $f$ is odd on $\mathbb{B}_{r}$ (i.e., $\left.f(-x)=-f(x), \forall x \in \mathbb{B}_{r}\right)$, then $\operatorname{deg}\left(f, \mathbb{B}_{r}, 0\right)$ is odd.

(6) Let $f(x)=A x-b$, with $A \in \mathbb{R}^{n \times n}$ being a nonsingular matrix and $b \in \mathbb{R}^{n}$. Then $\operatorname{deg}\left(f, A^{-1} b+\mathbb{B}_{r}, 0\right)=\operatorname{sgn}(\operatorname{det} A)= \pm 1$.

Let $V \in C^{1}\left(\mathbb{R}^{n} ; \mathbb{R}\right)$ and suppose that there exists $r_{0}>0$ such that, for every $r \geq r_{0}$, $0 \notin \nabla V\left(\partial \mathbb{B}_{r}\right)$. Then $\operatorname{deg}\left(\nabla V, \mathbb{B}_{r}, 0\right)$ is constant for $r \geq r_{0}$ and one defines the index of $V$ at infinity "ind $(V, \infty)$ " by

$$
\operatorname{ind}(V, \infty):=\operatorname{deg}\left(\nabla V, \mathbb{B}_{r}, 0\right), \quad \forall r \geq r_{0}
$$

Let us now recall some basic properties on convex functions defined on $\mathbb{R}^{n}$. Given a convex function $\varphi: \mathbb{R}^{n} \rightarrow \mathbb{R}$, it is well-known (see [19]) that

(a) $\varphi$ is continuous,

(b) For all $x \in \mathbb{R}^{n}$, the directional derivative of $\varphi$ at $x \in \mathbb{R}^{n}$ in the direction $\xi \in \mathbb{R}^{n}$, i.e.,

$$
\varphi^{\prime}(x ; \xi)=\lim _{\alpha \downarrow 0} \frac{\varphi(x+\alpha \xi)-\varphi(x)}{\alpha},
$$

exists and is finite for every $\xi \in \mathbb{R}^{n}$ (see, e.g., [10, p. 164]),

(c) For all $x \in \mathbb{R}^{n}$, the convex subdifferential of $\varphi$ at $x$ is a nonempty compact and convex subset of $\mathbb{R}^{n}$ and is defined by

$$
\partial \varphi(x)=\left\{w \in \mathbb{R}^{n}: \varphi(v)-\varphi(x) \geq\langle w, v-u\rangle, \forall v \in \mathbb{R}^{n}\right\}
$$


and

$$
w \in \partial \varphi(x) \Longleftrightarrow\langle w, \xi\rangle \leq \varphi^{\prime}(x ; \xi), \forall \xi \in \mathbb{R}^{n} .
$$

Since the subdifferetial operator is a maximal monotone operator (see for instance Brezis for the Hilbert setting and Rockafellar for the reflexive Banach setting), for each $\lambda>0$ the resolvent operator $J_{\lambda}^{\varphi}: \mathbb{R}^{n} \rightarrow \mathbb{R}^{n}$ defined by

$$
y \rightarrow J_{\lambda}^{\varphi}(y)=(I+\lambda \partial \varphi)^{-1}(y)
$$

is well-defined and is a contraction on $\mathbb{R}^{n}$, i.e.,

$$
\left\|J_{\lambda}^{\varphi}(x)-J_{\lambda}^{\varphi}(y)\right\| \leq\|x-y\|, \quad \forall x, y \in \mathbb{R}^{n}, \forall \lambda>0 .
$$

Hence $J_{\lambda}^{\varphi}$ is continuous on $\mathbb{R}^{n}$. For simplicity, we note $P_{\varphi}$ instead of $J_{1}^{\varphi}$ when the parameter $\lambda=1$.

\section{The Poincaré Operator}

Let us first recall some general existence and uniqueness result (see, e.g., [21]).

Theorem 3.1 Let $\varphi: \mathbb{R}^{n} \rightarrow \mathbb{R}$ be a convex function. Let $F: \mathbb{R}^{n} \rightarrow \mathbb{R}^{n}$ be a continuous operator such that, for some $\omega \in \mathbb{R}, F+\omega I$ is monotone, i.e.,

$$
\langle F(x)-F(y), x-y\rangle \geq-\omega\|x-y\|^{2}, \quad \forall x, y \in \mathbb{R}^{n} .
$$

Suppose that $f:[0,+\infty) \rightarrow \mathbb{R}^{n}$ satisfies

$$
f \in C^{0}\left([0,+\infty) ; \mathbb{R}^{n}\right), \quad \frac{\mathrm{d} f}{\mathrm{~d} t} \in L_{\mathrm{loc}}^{1}\left(0,+\infty ; \mathbb{R}^{n}\right) .
$$

Let $u_{0} \in \mathbb{R}^{n}$ and $0<T<+\infty$ be given. There exists a unique $u \in C^{0}\left([0, T] ; \mathbb{R}^{n}\right)$ such that

$$
\begin{aligned}
& \frac{\mathrm{d} u}{\mathrm{~d} t} \in L^{\infty}\left(0, T ; \mathbb{R}^{n}\right), \\
& u \text { is right-differentiable on }[0, T), \\
& u(0)=u_{0}, \\
& \frac{\mathrm{d} u}{\mathrm{~d} t}(t)+F(u(t))-f(t) \in-\partial \varphi(u(t)), \quad \text { a.e. } t \in[0, T] .
\end{aligned}
$$

Remark 3.1 Suppose that $F: \mathbb{R}^{n} \rightarrow \mathbb{R}^{n}$ is of the type

$$
F(x)=A x+\Psi^{\prime}(x)+F_{1}(x), \quad \forall x \in \mathbb{R}^{n},
$$

where $A \in \mathbb{R}^{n \times n}$ is a real matrix, $\Psi \in C^{1}\left(\mathbb{R}^{n} ; \mathbb{R}\right)$ is convex and $F_{1}$ is Lipschitz continuous, i.e.,

$$
\left\|F_{1}(x)-F_{1}(y)\right\| \leq k\|x-y\|, \quad \forall x, y \in \mathbb{R}^{n}
$$

for some constant $k>0$. Then $F$ is continuous and $F+\omega I$ is monotone provided that

$$
\omega \geq \sup _{\|x\|=1}\langle-A x, x\rangle+k .
$$

We note that if $F$ is $k$-Lipschitz, then $F+k I$ is monotone. 
Remark 3.2 Let $u:[0, T] \rightarrow \mathbb{R}$ be the unique solution of (3.1)-(3.4). Then using (3.4) and (2.1), we have

$$
\left\langle\frac{\mathrm{d} u}{\mathrm{~d} t}(t)+F(u(t))-f(t), \xi\right\rangle+\varphi^{\prime}(u(t) ; \xi) \geq 0, \quad \forall \xi \in \mathbb{R}^{n}, \quad \text { a.e. } t \in[0, T] .
$$

Let $T>0$ be given. Theorem 3.1 enables us to define the one parameter family $\{S(t): 0 \leq$ $t \leq T\}$ of operators from $\mathbb{R}^{n}$ into $\mathbb{R}^{n}$ as follows:

$$
\forall y \in \mathbb{R}^{n}, \quad S(t) y=u(t),
$$

$u$ being the unique solution on $[0, T]$ to the evolution problem (3.1)-(3.4). Note that

$$
\forall y \in \mathbb{R}^{n}, \quad S(0) y=y .
$$

Lemma 3.1 (See [21]) Let $T>0$ be given and let $a, b \in L^{1}(0, T ; \mathbb{R})$ with $b(t) \geq 0$, a.e. $t \in[0, T]$. Let the absolutely continuous function $w:[0, T] \rightarrow \mathbb{R}_{+}$satisfy

$$
(1-\alpha) \frac{\mathrm{d} w}{\mathrm{~d} t}(t) \leq a(t) w(t)+b(t) w^{\alpha}(t), \quad \text { a.e. } t \in[0, T]
$$

where $0 \leq \alpha<1$. Then

$$
w^{1-\alpha}(t) \leq w^{1-\alpha}(0) \mathrm{e}^{\int_{0}^{t} a(s) \mathrm{d} s}+\int_{0}^{t} \mathrm{e}^{\int_{s}^{t} a(q) \mathrm{d} q} b(s) \mathrm{d} s, \quad \forall t \in[0, T] .
$$

Theorem 3.2 Suppose that the assumptions of Theorem 3.1 hold. Then

$$
\|S(t) y-S(t) z\| \leq \mathrm{e}^{\omega t}\|y-z\|, \quad \forall y, z \in \mathbb{R}^{n}, t \in[0, T] .
$$

Proof Let $y, z \in \mathbb{R}^{n}$ be given. We have

$$
\begin{aligned}
-\left\langle\frac{\mathrm{d}}{\mathrm{d} t} S(t) y+F(S(t) y)-f(t), S(t) z-S(t) y\right\rangle-\varphi(S(t) z)+\varphi(S(t) y) \leq 0, & \text { a.e. } t \in[0, T], \\
\left\langle\frac{\mathrm{d}}{\mathrm{d} t} S(t) z+F(S(t) z)-f(t), S(t) z-S(t) y\right\rangle-\varphi(S(t) y)+\varphi(S(t) z) \leq 0, & \text { a.e. } t \in[0, T] .
\end{aligned}
$$

It results that

$$
\begin{aligned}
& \left\langle\frac{\mathrm{d}}{\mathrm{d} t}(S(t) z-S(t) y), S(t) z-S(t) y\right\rangle \\
\leq & \omega\|S(t) z-S(t) y\|^{2}-\langle[F+\omega I](S(t) z)-[F+\omega I](S(t) y), S(t) z-S(t) y\rangle, \quad \text { a.e. } t \in[0, T] .
\end{aligned}
$$

Our hypothesis ensures that $F+\omega I$ is monotone. It results that

$$
\frac{\mathrm{d}}{\mathrm{d} t}\|S(t) z-S(t) y\|^{2} \leq 2 \omega\|S(t) z-S(t) y\|^{2}, \quad \text { a.e. } t \in[0, T] .
$$

Using Lemma 3.1 with $w(\cdot):=\|S(\cdot) z-S(\cdot) y\|^{2}, a(\cdot):=2 \omega, b(\cdot)=0$ and $\alpha=0$, we get

$$
\|S(t) z-S(t) y\|^{2} \leq\|z-y\|^{2} \mathrm{e}^{2 \omega t}, \quad \forall t \in[0, T] .
$$

The conclusion follows.

Let us now consider the Poincaré operator $S(T): \mathbb{R}^{n} \rightarrow \mathbb{R}^{n} ; y \rightarrow S(T) y$. Theorem 3.2 ensures that $S(T)$ is Lipschitz continuous, i.e.,

$$
\|S(T) y-S(T) z\| \leq \mathrm{e}^{\omega T}\|y-z\|, \quad \forall y, z \in \mathbb{R}^{n} .
$$


Remark 3.3 (i) Note that if $F$ is continuous and monotone, then Theorem 3.2 holds with $\omega=0$. In this case, the Poincaré operator $S(T)$ is nonexpansive, i.e.,

$$
\|S(T) y-S(T) z\| \leq\|y-z\|, \quad \forall y, z \in \mathbb{R}^{n} .
$$

(ii) If $F$ is continuous and strongly monotone, i.e., there exists $\alpha>0$ such that

$$
\langle F(x)-F(y), x-y\rangle \geq \alpha\|x-y\|^{2}, \quad \forall x, y \in \mathbb{R}^{n},
$$

then Theorem 3.2 holds with $\omega=-\alpha<0$ and the Poincaré operator $S(T)$ is a contraction.

According to (3.5), the unique solution to the problem (3.1)-(3.4) satisfies, in addition, the periodicity condition

$$
u(0)=u(T)
$$

if and only if $y$ is a fixed point of $S(T)$, that is,

$$
S(T) y=y .
$$

Thus the problem of the existence of a periodic solution to the evolution problem (3.1)-(3.2) and (3.4) reduces to the existence of a fixed point for $S(T)$.

\section{Periodic Solutions}

Definition 4.1 Let $\Omega \subset \mathbb{R}^{n}$ be a given subset of $\mathbb{R}^{n}$. We say that $V \in C^{1}\left(\mathbb{R}^{n} ; \mathbb{R}\right)$ is a guiding function for (1.2) on $\Omega$ provided that

$$
\langle F(x)-f(t), \nabla V(x)\rangle+\varphi^{\prime}(x ; \nabla V(x))<0, \quad \forall x \in \Omega, t \in[0, T] .
$$

Remark 4.1 (i) Suppose that there exists a guiding function $V \in C^{1}\left(\mathbb{R}^{n} ; \mathbb{R}\right)$ for $(1.2)$ on $\partial \mathbb{B}_{r}(r>0)$, i.e.,

$$
\langle F(x)-f(t), \nabla V(x)\rangle+\varphi^{\prime}(x ; \nabla V(x))<0, \quad \forall x \in \partial \mathbb{B}_{r}, t \in[0, T] .
$$

Then for any $\tau \in[0, T]$, we have

$$
\operatorname{deg}\left(\nabla V, \mathbb{B}_{r}, 0\right)=(-1)^{n} \operatorname{deg}\left(\operatorname{id}_{\mathbb{R}^{n}}-P_{\varphi}\left(\operatorname{id}_{\mathbb{R}^{n}}-F+f(\tau)\right), \mathbb{B}_{r}, 0\right) .
$$

(ii) Suppose that there exists a guiding function $V \in C^{1}\left(\mathbb{R}^{n} ; \mathbb{R}\right)$ for $(1.2)$ on

$$
\Omega_{R}:=\left\{x \in \mathbb{R}^{n}:\|x\| \geq R\right\}=\mathbb{R}^{n} \backslash \mathbb{B}_{R}, \quad R>0 .
$$

Then for $r \geq R$ and any $\tau \in[0, T]$, we have

$$
\operatorname{ind}(V, \infty)=(-1)^{n} \operatorname{deg}\left(\operatorname{id}_{\mathbb{R}^{n}}-P_{\varphi}\left(\operatorname{id}_{\mathbb{R}^{n}}-F+f(\tau)\right), \mathbb{B}_{r}, 0\right) .
$$

Proposition 4.1 Suppose that there exists $R>0$ such that

$$
\langle F(x)-f(t), \nabla V(x)\rangle<0, \quad \forall x \in \mathbb{R}^{n},\|x\| \geq R, t \in[0, T] .
$$

Then for $r \geq R$ and any $\tau \in[0, T]$, we have

$$
\operatorname{ind}(V, \infty)=\operatorname{deg}\left(f(\tau)-F, \mathbb{B}_{r}, 0\right) .
$$


Proof Let $r \geq R$ be given and let $h:[0,1] \times \overline{\mathbb{B}}_{r} \rightarrow \mathbb{R}^{n},(\lambda, y) \rightarrow h(\lambda, y):=\lambda \nabla V(y)+$ $(1-\lambda)(f(0)-F(y))$. We claim that $h(\lambda, y) \neq 0, \forall y \in \partial \mathbb{B}_{r}, \lambda \in[0,1]$. Indeed, suppose by contradiction that

$$
\lambda \nabla V(y)+(1-\lambda)(f(\tau)-F(y))=0
$$

for some $y \in \partial \mathbb{B}_{r}$ and $\lambda \in[0,1]$. Then

$$
\lambda\langle\nabla V(y), f(\tau)-F(y)\rangle=-(1-\lambda)\|f(\tau)-F(y)\|^{2} .
$$

Obviously $\lambda \neq 0$. Indeed, if $\lambda=0$, we obtain $f(\tau)-F(y)=0$, a contradiction to condition (4.3) since $y \in \partial \mathbb{B}_{r}$ and $r \geq R$. Thus (4.4) yields $\langle\nabla V(y), f(\tau)-F(y)\rangle<0$ which also contradicts (4.3). Thus $\operatorname{deg}\left(\nabla V, \mathbb{B}_{r}, 0\right)=\operatorname{deg}\left(h(1, \cdot), \mathbb{B}_{r}, 0\right)=\operatorname{deg}\left(h(0, \cdot), \mathbb{B}_{r}, 0\right)=\operatorname{deg}\left(f(\tau)-F, \mathbb{B}_{r}, 0\right)$.

Theorem 4.1 We make the assumptions that $f \in C^{0}\left([0,+\infty) ; \mathbb{R}^{n}\right)$ and $\frac{\mathrm{d} f}{\mathrm{~d} t} \in L_{\mathrm{loc}}^{1}(0,+\infty$; $\left.\mathbb{R}^{n}\right)$. Let $\varphi: \mathbb{R}^{n} \rightarrow \mathbb{R}$ be a convex function. Let $F: \mathbb{R}^{n} \rightarrow \mathbb{R}^{n}$ be a mapping such that $F+\omega I$ is monotone for some $\omega \in \mathbb{R}$. Suppose that there exist constants $C_{1} \geq 0$ and $C_{2} \geq 0$ such that

$$
\langle F(x), x\rangle+\varphi^{\prime}(x ; x) \leq C_{1}\|x\|^{2}+C_{2}\|x\|, \quad \forall x \in \mathbb{R}^{n} .
$$

Let $T>0$ be given. Assume that there exists a (guiding) function $V \in C^{1}\left(\mathbb{R}^{n} ; \mathbb{R}\right)$ and $R>0$ such that

$$
\langle F(x)-f(t), \nabla V(x)\rangle+\varphi^{\prime}(x ; \nabla V(x))<0, \quad \forall x \in \mathbb{R}^{n},\|x\| \geq R, t \in[0, T] .
$$

Then there exists at least one $u \in C^{0}\left([0, T] ; \mathbb{R}^{n}\right)$ such that $\frac{\mathrm{d} u}{\mathrm{~d} t} \in L^{\infty}\left(0, T ; \mathbb{R}^{n}\right)$,

$$
\begin{aligned}
& u(0)=u(T), \\
& \frac{\mathrm{d} u}{\mathrm{~d} t}(t)+F(u(t))-f(t) \in-\partial \varphi(u(t)), \quad \text { a.e. } t \in[0, T] .
\end{aligned}
$$

Proof We will prove that there exists $r_{0}>R$ such that for any $\tau \in[0, T]$ we have

$$
\begin{aligned}
\operatorname{deg}\left(\operatorname{id}_{\mathbb{R}^{n}}-S(T), \mathbb{B}_{r}, 0\right) & =\operatorname{deg}\left(\operatorname{id}_{\mathbb{R}^{n}}-P_{\varphi}\left(\operatorname{id}_{\mathbb{R}^{n}}-F+f(\tau)\right), \mathbb{B}_{r}, 0\right) \\
& =(-1)^{n} \operatorname{ind}(V, \infty), \quad \forall r \geq r_{0} .
\end{aligned}
$$

Let us first remark that without loss of generality, we may assume $C_{1}>0$. We set

$$
r_{0}:=R \mathrm{e}^{C_{1} T}+\frac{C_{2}}{C_{1}}\left(\mathrm{e}^{C_{1} T}-1\right)+\int_{0}^{T}\|f(s)\| \mathrm{e}^{C_{1} s} \mathrm{~d} s .
$$

Step 1 We claim that if $y \in \mathbb{R}^{n},\|y\|=r$ with $r \geq r_{0}$, then

$$
\|S(t) y\| \geq R, \quad \forall t \in[0, T] .
$$

Suppose by contradiction that there exists $t^{*} \in[0, T]$ such that $\left\|S\left(t^{*}\right) y\right\|<R$. We know that $u(\cdot) \equiv S(\cdot) y$ satisfies

$$
\frac{\mathrm{d} u}{\mathrm{~d} t}(t)+F(u(t))-f(t) \in-\partial \varphi(u(t)), \quad \text { a.e. } t \in[0, T],
$$


and thus

$$
\frac{\mathrm{d} u}{\mathrm{~d} t}\left(t^{*}-t\right)+F\left(u\left(t^{*}-t\right)\right)-f\left(t^{*}-t\right) \in-\partial \varphi\left(u\left(t^{*}-t\right)\right), \quad \text { a.e. } t \in\left[0, t^{*}\right] .
$$

Setting

$$
Y(t)=u\left(t^{*}-t\right), \quad t \in\left[0, t^{*}\right]
$$

we derive

$$
-\frac{\mathrm{d} Y}{\mathrm{~d} t}(t)+F(Y(t))-f\left(t^{*}-t\right) \in-\partial \varphi(Y(t)), \quad \text { a.e. } t \in\left[0, t^{*}\right] .
$$

Thus

$$
\left\langle\frac{\mathrm{d} Y}{\mathrm{~d} t}(t), \xi\right\rangle \leq\left\langle F(Y(t))-f\left(t^{*}-t\right), \xi\right\rangle+\varphi^{\prime}(Y(t) ; \xi), \quad \forall \xi \in \mathbb{R}^{n}, \quad \text { a.e. } t \in\left[0, t^{*}\right] .
$$

For $\xi=Y(t)$, we have

$$
\begin{aligned}
\left\langle\frac{\mathrm{d} Y}{\mathrm{~d} t}(t), Y(t)\right\rangle & \leq\left\langle F(Y(t))-f\left(t^{*}-t\right), Y(t)\right\rangle+\varphi^{\prime}(Y(t) ; Y(t)) \\
& \leq C_{1}\|Y(t)\|^{2}+C_{2}\|Y(t)\|+\left\|f\left(t^{*}-t\right)\right\|\|Y(t)\|, \quad \text { a.e. } t \in\left[0, t^{*}\right] .
\end{aligned}
$$

Thus

$$
\frac{1}{2} \frac{\mathrm{d}}{\mathrm{d} t}\|Y(t)\|^{2} \leq C_{1}\|Y(t)\|^{2}+\left(C_{2}+\left\|f\left(t^{*}-t\right)\right\|\right)\|Y(t)\|, \quad \text { a.e. } t \in\left[0, t^{*}\right] .
$$

Using Lemma 3.1 with $\alpha:=\frac{1}{2}, w(\cdot):=\|Y(\cdot)\|^{2}, a(\cdot):=C_{1}$ and $b(\cdot):=C_{2}+\left\|f\left(t^{*}-\cdot\right)\right\|$, we obtain

$$
\|Y(t)\| \leq\|Y(0)\| \mathrm{e}^{C_{1} t}+\int_{0}^{t} C_{2} \mathrm{e}^{C_{1}(t-s)} \mathrm{d} s+\int_{0}^{t}\left\|f\left(t^{*}-s\right)\right\| \mathrm{e}^{C_{1}(t-s)} \mathrm{d} s, \quad \forall t \in\left[0, t^{*}\right] .
$$

Since $Y\left(t^{*}\right)=u(0)=S(0) y=y$ and $Y(0)=u\left(t^{*}\right)=S\left(t^{*}\right) y$, we get

$$
\begin{aligned}
\|y\| & \leq\left\|S\left(t^{*}\right) y\right\| \mathrm{e}^{C_{1} t^{*}}+\int_{0}^{t^{*}} C_{2} \mathrm{e}^{C_{1}\left(t^{*}-s\right)} \mathrm{d} s+\int_{0}^{t^{*}}\left\|f\left(t^{*}-s\right)\right\| \mathrm{e}^{C_{1}\left(t^{*}-s\right)} \mathrm{d} s \\
& <R \mathrm{e}^{C_{1} T}+\frac{C_{2}}{C_{1}}\left(\mathrm{e}^{C_{1} T}-1\right)+\int_{0}^{T}\|f(s)\| \mathrm{e}^{C_{1} s} \mathrm{~d} s=r_{0} .
\end{aligned}
$$

Hence, $\|y\|<r_{0}$, a contradiction.

Step 2 Let $r \geq r_{0}$ be given. We claim that there exist $\varepsilon>0$ and $T^{*} \in(0, T]$ such that

$$
\begin{aligned}
& \langle F(x)-f(t), \nabla V(y)\rangle+\varphi^{\prime}(x ; \nabla V(y))<0, \\
& \forall x \in \mathbb{R}^{n}, y \in \mathbb{R}^{n},\|y\|=r,\|x-y\| \leq \varepsilon, t \in\left[0, T^{*}\right] .
\end{aligned}
$$

Indeed, recalling that the mapping $(z, \xi) \mapsto \varphi^{\prime}(z ; \xi)$ is upper semicontinuous (see, e.g., [10]), we note that the mapping $(t, x, y) \mapsto\langle F(x)-f(t), \nabla V(y)\rangle+\varphi^{\prime}(x ; \nabla V(y))$ is upper semicontinuous on $[0, T] \times \mathbb{R}^{n} \times \mathbb{R}^{n}$ and if $y \in \mathbb{R}^{n}$ and $\|y\|=r \geq r_{0} \geq R$, then (by condition (4.6))

$$
\langle F(y)-f(0), \nabla V(y)\rangle+\varphi^{\prime}(y ; \nabla V(y))<0 .
$$

Thus, for $t>0$ close to 0 , let us say $t \leq T^{*}$; and $x$ close to $y$, let us say $\|x-y\| \leq \varepsilon, \varepsilon>0$, small; we have $\langle F(x)-f(0), \nabla V(y)\rangle+\varphi^{\prime}(x ; \nabla V(y))<0$. 
Step 3 We claim that there exists $\bar{T} \in\left(0, T^{*}\right]$ such that

$$
\|S(t) y-y\| \leq \varepsilon, \quad \forall y \in \partial \mathbb{B}_{r}, \forall t \in[0, \bar{T}] .
$$

Indeed, by contradiction, suppose that there exist sequences $t_{n} \in\left[0, \frac{T^{*}}{n}\right](n \in \mathbb{N}, n \geq 1)$ and $y_{n} \in \mathbb{R}^{n},\left\|y_{n}\right\|=r$, such that $\left\|S\left(t_{n}\right) y_{n}-y_{n}\right\|>\varepsilon$. Taking a subsequence if necessary, we may assume that $t_{n} \rightarrow 0+$ and $y_{n} \rightarrow y^{*} \in \partial \mathbb{B}_{r}$. On the other hand, we have

$$
\begin{aligned}
\left\|S\left(t_{n}\right) y_{n}-y_{n}\right\| & =\left\|S\left(t_{n}\right) y_{n}-S\left(t_{n}\right) y^{*}+S\left(t_{n}\right) y^{*}-y_{n}\right\| \\
& \leq\left\|S\left(t_{n}\right) y_{n}-S\left(t_{n}\right) y^{*}\right\|+\left\|S\left(t_{n}\right) y^{*}-y_{n}\right\| .
\end{aligned}
$$

Then using Theorem 3.2, we obtain

$$
\left\|S\left(t_{n}\right) y_{n}-y_{n}\right\| \leq \sqrt{\mathrm{e}^{2 w t_{n}}}\left\|y_{n}-y^{*}\right\|+\left\|S\left(t_{n}\right) y^{*}-y_{n}\right\| .
$$

Using the continuity of the map $t \mapsto S(t) y$, we see that $\left\|S\left(t_{n}\right) y_{n}-y_{n}\right\| \rightarrow 0$, a contradiction.

Step 4 Let $H_{\bar{T}}:[0,1] \times \bar{C}_{r} \rightarrow \mathbb{R}^{n},(\lambda, y) \rightarrow H_{\bar{T}}(\lambda, y):=y-(1-\lambda) \nabla V(y)-S(\lambda \bar{T}) y$. We claim that the homotopy $H_{\bar{T}}$ is such that $0 \neq H_{\bar{T}}(\lambda, y), \forall y \in \partial \mathbb{B}_{r}, \lambda \in[0,1]$. By contradiction, suppose that there exists $y \in \mathbb{R}^{n},\|y\|=r$ and $\lambda \in[0,1]$ such that

$$
y-(1-\lambda) \nabla V(y)-S(\lambda \bar{T}) y=0 .
$$

Then

$$
S(\lambda \bar{T}) y-y=-(1-\lambda) \nabla V(y)
$$

and thus

$$
\langle S(\lambda \bar{T}) y-y, \nabla V(y)\rangle=-(1-\lambda)\|\nabla V(y)\|^{2} \leq 0 .
$$

On the other hand, we know that

$$
\begin{aligned}
& \left\langle\frac{\mathrm{d}}{\mathrm{d} t} S(t) y, v-S(t) y\right\rangle+\varphi(v)-\varphi(S(t) y) \\
\geq & \langle-F(S(t) y)+f(t), v-S(t) y\rangle, \quad \forall v \in \mathbb{R}^{n}, \quad \text { a.e. } t \in[0, T] .
\end{aligned}
$$

Thus

$$
\left\langle\frac{\mathrm{d}}{\mathrm{d} t} S(t) y, \nabla V(y)\right\rangle+\varphi^{\prime}(S(t) y ; \nabla V(y)) \geq\langle-F(S(t) y)+f(t), \nabla V(y)\rangle, \quad \text { a.e. } t \in[0, T] .
$$

Therefore,

$$
\left\langle\int_{0}^{\lambda \bar{T}} \frac{\mathrm{d}}{\mathrm{d} s} S(s) y \mathrm{~d} s, \nabla V(y)\right\rangle \geq \int_{0}^{\lambda \bar{T}}\langle-F(S(s) y)+f(s), \nabla V(y)\rangle-\varphi^{\prime}(S(s) y ; \nabla V(y)) \mathrm{d} s .
$$

Step 1 of this proof ensures that $\|S(t) y\| \geq R, \forall t \in[0, \lambda \bar{T}] \subset[0, T]$. Step 3 of this proof guarantees that $\|S(t) y-y\| \leq \varepsilon, \forall t \in[0, \lambda \bar{T}] \subset[0, \bar{T}]$. Then using Step 2 of this proof, we may assert that the map $s \mapsto\langle F(S(s) y)-f(s), \nabla V(y)\rangle+\varphi^{\prime}(S(s) y ; \nabla V(y))$ is upper semicontinuous and strictly negative on $[0, \lambda \bar{T}]$. Thus

$$
\int_{0}^{\lambda \bar{T}}\langle-F(S(s) y)+f(s), \nabla V(y)\rangle-\varphi^{\prime}(S(s) y ; \nabla V(y)) \mathrm{d} s>0 .
$$


We obtain

$$
\langle S(\lambda \bar{T}) y-y, \nabla V(y)\rangle=\left\langle\int_{0}^{\lambda \bar{T}} \frac{\mathrm{d}}{\mathrm{d} s} S(s) y \mathrm{~d} s, \nabla V(y)\right\rangle>0 .
$$

This contradicts relation $(4.12)$.

Step 5 Thanks to Step 4 of this proof, we may use the invariance by homotopy property of the topological degree and see that

$$
\begin{aligned}
\operatorname{deg}\left(\operatorname{id}_{\mathbb{R}^{n}}-S(\bar{T}), \mathbb{B}_{r}, 0\right) & =\operatorname{deg}\left(H_{\bar{T}}(1, \cdot), \mathbb{B}_{r}, 0\right) \\
& =\operatorname{deg}\left(H_{\bar{T}}(0, \cdot), \mathbb{B}_{r}, 0\right) \\
& =\operatorname{deg}\left(-\nabla V, \mathbb{B}_{r}, 0\right) \\
& =(-1)^{n} \operatorname{deg}\left(\nabla V, \mathbb{B}_{r}, 0\right) .
\end{aligned}
$$

Step 6 Let $H:[0,1] \times \overline{\mathbb{B}}_{r} \rightarrow \mathbb{R}^{n},(\lambda, y) \rightarrow H(\lambda, y):=y-S((1-\lambda) T+\lambda \bar{T}) y$. We claim that $H(\lambda, y) \neq 0, \forall y \in \partial \mathbb{B}_{r}, \lambda \in[0,1]$. By contradiction, suppose that there exists $y \in \mathbb{R}^{n}$, $\|y\|=r$ and $\lambda \in[0,1]$ such that $y=S((1-\lambda) T+\lambda \bar{T}) y$. Set $h:=(1-\lambda) T+\lambda \bar{T}$. We have

$$
y=S(h) y
$$

and thus

$$
V(y)=V(S(h) y)
$$

On the other hand,

$$
\begin{aligned}
& \left\langle\frac{\mathrm{d}}{\mathrm{d} t} S(t) y, v-S(t) y\right\rangle+\varphi(v)-\varphi(S(t) y) \\
\geq & \langle-F(S(t) y)+f(t), v-S(t) y\rangle, \quad \forall v \in \mathbb{R}^{n}, \quad \text { a.e. } t \in[0, T] .
\end{aligned}
$$

Thus

$$
\begin{aligned}
& \left\langle\frac{\mathrm{d}}{\mathrm{d} t} S(t) y, \nabla V(S(t) y)\right\rangle+\varphi^{\prime}(S(t) y ; \nabla V(S(t) y)) \\
\geq & \langle-F(S(t) y)+f(t), \nabla V(S(t) y)\rangle, \quad \text { a.e. } t \in[0, T] .
\end{aligned}
$$

Step 1 of this proof ensures that $\|S(t) y\| \geq R, \forall t \in[0, T]$. The map $s \rightarrow\langle F(S(s) y)-$ $f(s), \nabla V(S(s) y)\rangle+\varphi^{\prime}(S(s) y ; \nabla V(S(s) y))$ is upper semicontinuous and (by condition (4.6)) strictly negative on $[0, T]$. Thus, using (4.16), we obtain

$$
\begin{aligned}
V(S(h) y)-V(y) & =\int_{0}^{h} \frac{\mathrm{d}}{\mathrm{d} s} V(S(s) y) \mathrm{d} s \\
& =\int_{0}^{h}\left\langle\frac{\mathrm{d}}{\mathrm{d} s} S(s) y, \nabla V(S(s) y)\right\rangle \\
& \geq \int_{0}^{h}\langle-F(S(s) y)+f(s), \nabla V(S(s) y)\rangle-\varphi^{\prime}(S(s) y ; \nabla V(S(s) y)) \mathrm{d} s \\
& >0 .
\end{aligned}
$$

This is a contradiction to (4.14). 
Step 7 Thanks to Step 6 of this proof, we may use the invariance by homotopy property of the topological degree and see that

$$
\begin{aligned}
\operatorname{deg}\left(\operatorname{id}_{\mathbb{R}^{n}}-S(T), \mathbb{B}_{r}, 0\right) & =\operatorname{deg}\left(H(0, \cdot), \mathbb{B}_{r}, 0\right) \\
& =\operatorname{deg}\left(H(1, \cdot), \mathbb{B}_{r}, 0\right) \\
& =\operatorname{deg}\left(\operatorname{id}_{\mathbb{R}^{n}}-S(\bar{T}), \mathbb{B}_{r}, 0\right) .
\end{aligned}
$$

In conclusion, for all $r \geq r_{0}$, we have

$$
\begin{aligned}
& \operatorname{deg}\left(\operatorname{id}_{\mathbb{R}^{n}}-S(T), \mathbb{B}_{r}, 0\right)=\operatorname{deg}\left(\operatorname{id}_{\mathbb{R}^{n}}-S(\bar{T}), \mathbb{B}_{r}, 0\right), \\
& \operatorname{deg}\left(\operatorname{id}_{\mathbb{R}^{n}}-S(\bar{T}), \mathbb{B}_{r}, 0\right)=(-1)^{n} \operatorname{deg}\left(\nabla V, \mathbb{B}_{r}, 0\right) .
\end{aligned}
$$

Thus

$$
\operatorname{deg}\left(\operatorname{id}_{\mathbb{R}^{n}}-S(T), \mathbb{B}_{r}, 0\right)=(-1)^{n} \operatorname{ind}(V, \infty) .
$$

Finally, for any $\tau \in[0, T]$, we also have (see Remark 4.1)

$$
(-1)^{n} \operatorname{ind}(V, \infty)=\operatorname{deg}\left(\operatorname{id}_{\mathbb{R}^{n}}-P_{\varphi}\left(\operatorname{id}_{\mathbb{R}^{n}}-F+f(\tau)\right), \mathbb{B}_{r}, 0\right) .
$$

It results that, for $r>0$ large enough, we have $\operatorname{deg}\left(\operatorname{id}_{\mathbb{R}^{n}}-S(T), \mathbb{B}_{r}, 0\right) \neq 0$ and the existence of a fixed point for the Poincaré operator follows from the existence property of the topological degree.

\section{References}

[1] Adly, S., Goeleven, D. and Théra, M., A continuation method for a class of periodic evolution variational inequalities, Chin. Ann. Math., 28B(6), 2007, 629-650.

[2] Baiocchi, C. and Capelo, A., Variational and Quasi-variational Inequalities: Applications to Free Boundary Problems, John Wiley and Sons, New York, 1984.

[3] Brezis, H. and Stampacchia, G., Sur la régularité de la solution d'inéquations elliptiques, Bull. Soc. Math. France, 96, 1968, 153-180.

[4] Brezis, H., Problèmes unilatéraux, J. Math. Pures Appl., 51, 1972, 1-168.

[5] Brezis, H., Opérateurs Maximaux Monotones et Semi-groupes de Contractions dans les Espaces de Hilbert, North-Holland, Amsterdam, 1973.

[6] Cottle, R. W., Nonlinear Programs with Positively Bounded Jacobians, Ph.D Thesis, University of California at Berkley, 1964.

[7] Facchinei, F. and Pang, J.-S., Finite Dimensional Variational Inequalities and Complementarity Problems, Springer-Verlag, New York, 2003.

[8] Giannessi, F., Constrained Optimization and Image Space Analysis, Springer-Verlag, New York, 2005.

[9] Glowinski, R., Lions, J. L. and Trémolières, R., Analyse numérique des inéquations variationnelles, Tome 1: Théorie générale premières applications, Méthodes Mathématiques de l'Informatique, Vol. 5, Dunod, Paris, 1976.

[10] Hiriart-Urruty, J. B. and Lemaréchal, C., Fundamentals of Convex Analysis, Springer-Verlag, Berlin, 1993.

[11] Krasonel'skii, M. A., The Operator of Translation along the Trajectories of Differential Equations (in Russian), Nauka, Moscow, 1966; Translations of Mathematical Monographs, Vol. 19, A. M. S., Providence, RI, 1968.

[12] Kinderlehrer, D. and Stampacchia, G., An Introduction to Variational Inequalities and Their Applications, Academic Press, New York, 1980.

[13] Krasonel'skii, M. A. and Zabreiko, P. P., Geometrical Methods of Nonlinear Analysis (in Russian), Nauka, Moscow, 1975; Springer-Verlag, Berlin, 1984. 
[14] Lions, J. L., The work of G. Stampacchia in variational inequalities, Variational Inequalities and Complementarity Problems, Wiley, Chichester, 1980, 1-24; Variational Analysis and Applications, Nonconvex Optim. Appl., 79, Springer-Verlag, New York, 2005, 3-30.

[15] Lions, J. L. and Stampacchia, G., Inéquation variationnelles non coercives, C. R. Acad. Sci., 261, 1965, $25-27$.

[16] Lloyd, N. G., Degree Theory, Cambridge University Press, Cambridge, 1978.

[17] Mawhin, J., Continuation theorems and periodic solutions of ordinary differential equations, Topological Methods in Differential Equations and Inclusions, A. Granas, M. Frigon and G. Sabidussi (eds.), NATO Sci. Ser. C, Math. Phys. Sci., 472, Kluwer Academic Publishers, Dordrecht, 1994.

[18] Robinson, S. M., Generalized equations and their solutions, part I: Basic theory, Math. Progr. Study, 10, 1979, 128-141.

[19] Rockafellar, R. T., Convex Analysis, Princeton University Press, Princeton, 1968.

[20] Stampacchia, G., Formes bilinéaires coercitives sur les ensembles convexes, C. R. Acad. Sci., 258, 1964, 4413-4416.

[21] Showalter, R. E., Monotone Operators in Banach Spaces and Nonlinear Partial Differential Equations, Mathematical Surveys and Monographs, Vol. 49, A. M. S., Providence, RI, 1997. 